\title{
Interference Pricing based Resource Allocation for D2D Communications in Cellular Networks
}

\author{
Xiaomeng Li, and Tiejun Lv* \\ School of Information and Communication Engineering \\ Beijing University of Posts and Telecommunications, Beijing, China 100876 \\ [e-mail: \{lvtiejun, lixiaomeng1011\}@bupt.edu.cn] \\ *Corresponding author: Tiejun Lv
}

Received March 5, 2017; revised November 5, 2017; accepted March 26, 2018;

published September 30, 2018

\begin{abstract}
Abstract---We consider the Device-to-Device (D2D) communications in cellular networks where each cellular user (CU) shares the same resource with multiple D2D users (DUs). In this paper, we aim to maximize the energy efficiency (EE) of the D2D networks, subject to an interference constraint required by the CU. Since the cellular and D2D communications belong to different networks, we consider to incentivize base station (BS) while assisting the DUs. To this end, we propose a Stackelberg game based interference pricing framework for the considered D2D communications in cellular networks. Unlike most of the existing methods, we use interference pricing framework to jointly address the EE resource allocation problem and the interference management in our networks rather than only improve the EE of the DUs or protect cellular networks. In particular, BS and all the users do not need all channel state information, which is more realistic in practice. In addition, two different pricing strategies are also proposed. Based on the two strategies, we analyze the equilibrium of the game. Moreover, in the first strategy, the upper and lower boundaries of the interference price are obtained. The closed-form expression is gained with a backward induction for the second strategy. Both offer valuable insights to the considered scenarios. Finally, compared with the existing work, the EE of the D2D communications is significantly improved. The advantageous performance of our scheme are demonstrated by the simulation results.
\end{abstract}

Keywords: Energy efficiency, interference pricing framework, resource allocation, Stackelberg game.

This work is supported by the National Natural Science Foundation of China (NSFC) (Grant No. 61671072). 


\section{Introduction}

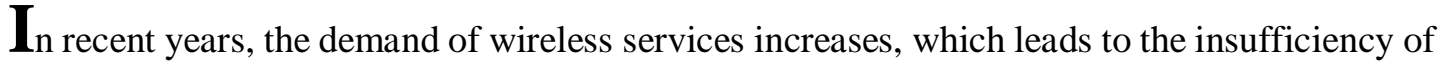
available spectrum resource. In order to meet such increasing demands, Device-to-Device (D2D) communications are considered as promising techniques [1]. For the D2D users (DUs), they can communicate to each other directly without the information exchange at base stations (BSs). D2D communications have potential to significantly improve network capacity and save power consumption [2]. Moreover, facing the high energy consumption of the heterogeneous networks, D2D becomes an important technology to improve the energy efficiency. However, because D2D and cellular communications share the same resource, cross-tier interference between the cellular and D2D communications might significantly degrade communication performance without proper interference management.

Interference management mainly includes two types, i.e., power allocation strategies and interference mitigation/cancelation strategies. Various interference control methods have been proposed to alleviate the interference within the D2D communications underlaying cellular networks. In particular, an opportunistic interference alignment scheme has been proposed for a multi-way relay network by mitigating the interference [3]. However, plenty of antennas are required for the equipment in this scheme. Then, in [4], an interference limited areas based power allocation scheme is employed in the heterogeneous networks to mitigate the interference. Only the DUs in the interference limited area can share the spectrum with the cellular users (CUs). According to [3], [4], the data rates of CUs can be guaranteed. However, due to the interference power control, the DUs in the interference limited areas cannot be close to the corresponding CU. Then, due to the path loss, the energy efficiency (EE), defined as the transmitted bits per unit energy consumption, for the D2D communications would be low. EE has always been a major concern. Inspired by previous works, in [5], the authors use the cluster to achieve high EE. DUs that share content form cooperative clusters. Then, the received data from the BS at one DU is multicast to other DUs within the cluster at a short distance. A power control strategy with different resource sharing modes between cellular and D2D networks has been presented in [6]. In the work mentioned earlier, the authors mainly improve EE based on the power allocation. It should be noted that, in our considered scenario, there are two different networks, i.e., D2D and cellular networks. When we consider the EE of D2D cellular networks, the communication quality of the CUs is also an important concern. In order to improve EE of the D2D communications and control the interference in CUs caused by DUs, we shall jointly optimize EE of DUs and interference power constraint strategies for D2D communications. Also, the joint control problem referred above is difficult and non-convex.

Aiming to solve the joint control problem, we introduce game framework in [7] which can model the interaction between DUs and BS. When DUs get the benefits from the cellular networks by sharing the resource, the performance of the cellular networks would be affected by these DUs. To this end, each DU should pay a certain amount for the interference caused to the CU. Motivated by this observation, interference pricing mechanisms for resource management in wireless networks have received considerable attention recently [8]. In previous studies with pricing mechanisms, most existing schemes mainly focus on how to maximize the throughput but ignore the EE, such as [9], [10]. In the D2D communications, DUs call for high EE. However, CUs also should be protected. Furthermore, the schemes in [9], [10] are only applicable to the single co-channel D2D scenario, which might not be able to 
address the interference management in the more general scenario. Thus, these points motivate our work in this paper.

In this paper, we consider the D2D communications underlaying cellular networks, in which each CU shares the same resource block with multiple D2D links. We assume that the spectrums used by CUs are orthogonal each other, and each CU only obtains interference from the corresponding DUs who share the same resource. Without loss of generality, one CU is only considered in the system model. To the best of our knowledge, our proposed method using pricing based Stackberg game to jointly solve the EE maximization problem and the interference constraint has not been investigated in our considered networks. In particular, the key contributions of our work are listed as follows:

1) Aiming to improve the EE of the DUs while controlling the interference caused by DUs, we propose a Stackelberg game based interference pricing scheme to jointly address the interference management and EE optimization in the considered system. In particular, in order to simplify the optimization problem, we introduce the EE expected capacity in [11] which is different from the pure rate function. Especially, BS and all users can make decisions with local channel state information (CSI). In the Stackelberg game, BS and DUs are modelled as seller and buyer, respectively. BS takes into consideration the behavior of the DUs, and then sets the interference prices for the DUs to maximize the profit under a maximum interference tolerance margin. After that, the DUs would compete for the maximum net utility in consideration of the interference constraint.

2) In addition, two pricing strategies, i.e., uniform pricing and uneven pricing strategies are proposed and analyzed. Regarding the uniform pricing strategy, the lower and upper boundaries of the prices can be obtained. Then, it would be easier for us to know how to set the initial interference price. For the uneven pricing strategy, a backward induction is used to find the solution of the Stackelberg equilibrium. Then, the closed-form expression can be obtained. According to the analysis, we can decide whether DUs can be admitted in the cellular networks. Numerical simulations show that the performance of our scheme can outperform the existing methods, and our scheme can control the interference and improve the EE effectively.

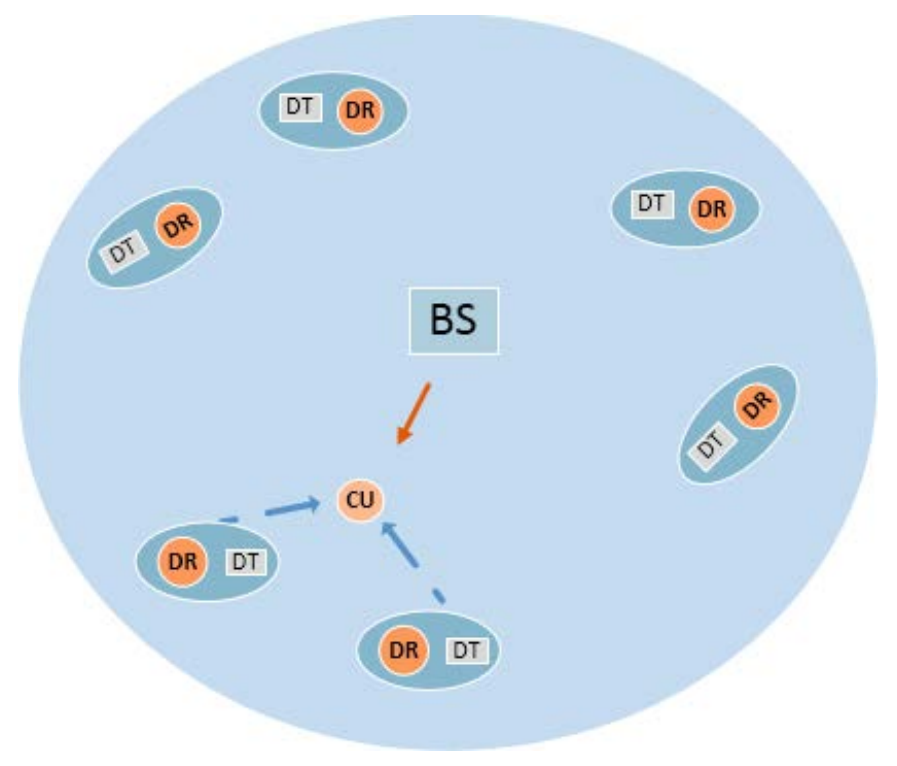

Fig. 1. D2D Communications Underlaying Cellular Networks. 
The rest of the paper is organized as follows. In Section 2, we specify the system model. In Section 3, we propose the Stackelberg game-based scheme. In Section 4, the numerical results are shown with discussion. Finally, we conclude in Section 5.

\section{System Model}

In this section, we consider the D2D communications underlaying cellular networks, in which one CU and $N$ D2D share the same resource block. As shown in Fig. 1, in each DU, there is one transmitter (DT) and one receiver (DR). In order to protect the quality of service (QoS) of the $\mathrm{CU}$, we need to propose a scheme to guarantee that interference power received from the D2D networks should not be larger than $\Upsilon$. The maximum transmission power of the DUs is $p_{\max }$.

The interference power received at the CU from the D2D networks can be denoted as

$$
I_{m}=\sum_{n=1}^{N} p_{n} f_{n m},
$$

where $p_{n}$ is the transmission power of the $n$-th DU, $f_{n m}$ is the channel gain between the $n$-th DT and the CU.

In consideration of the cost of transmission, the energy efficient expected capacity expression [11] of the $n$-th DU can be expressed as

$$
R_{n}=W \log \left(1+\frac{p_{n} g_{n}}{\sigma_{n}^{2}+\sum_{l \neq n}^{N} p_{l} h_{l n}+p_{B} g_{n m}}\right)-x p_{n},
$$

where $W$ is the bandwidth of the channel, and $\sigma_{n}^{2}$ is the noise power, $g_{n}$ is the channel gain between the $n$-th D2D pairs, $h_{l n}$ is the channel gain between the $l$-th DT and the $n$-th DR, $p_{B}$ is the transmission power of the BS, $g_{n m}$ is the channel gain between the BS and the $n$-th DR. We can view this expression as a direct utility function whose second term can be seen as the cost on transmit factor. Then, $x$ represents the normalized average cost per unit power for the DU to allocate resource and the unit of $x$ is bits/s/W $[11,12,13]$.

\section{THE STACKELBERG GAME-BASED SCHEME}

\subsection{A Brief Review of the Stackelberg Game}

Stackelberg game is a leader-follower game. One player acts as a leader and the rest as followers. Each participant strives for the best interests under certain conditions. The basic idea of the game is to find an optimal strategy for the leader, assuming that the followers react in such a rational way that they optimize their objective functions in consideration of the leader's actions [14].

In our considered networks, because the DUs get benefit by sharing the same resource with CUs and ignore the decline in performance of CUs, DUs need to pay for the interference caused to the CUs. In this paper, we incentivize BS to assist the DUs based on interference pricing framework in which BS provide the interference price. As DUs also call for high EE, the net utility function of DUs equals to EE expected capacity minus interference cost paid to BS. As for BS, it would obtain the interference profit. So, the net utility function of BS should be the profit of BS. Then, the optimization problem of maximizing the EE, subject to an 
interference constraint in our scenario can be analyzed by using Stackelberg game, where the BS can be regarded as the leader, i.e., seller and the DUs can be regarded as the followers, i.e., buyers. BS offers the interference prices to the DUs according to the interference caused by the DUs, then, based on the action of the BS and the interference prices provided by BS, DUs need to adjust their transmission power to maximize the EE.

\subsection{Stackelberg Game Definition}

In the Stackelberg game, we use an interference pricing framework, where the relations between resource demand and supply can be reflected.

\section{1) The D2D (Buyer) Side}

For the D2D networks, the net utility function for the $n$-th DU can be written as

$$
\begin{gathered}
\Psi_{n}\left(p_{n}\right)=W \log \left(1+\frac{p_{n} g_{n}}{\sigma_{n}^{2}+\sum_{l \neq n}^{N} p_{l} h_{l n}+p_{B} g_{n m}}-\mu_{n} x p_{n}-\varepsilon_{n} p_{n} f_{n m} y_{n},\right. \\
\text { s.t. } 0 \leq p_{n} \leq p_{\max },
\end{gathered}
$$

where $\varepsilon_{n}$ is the weight to balance the data rate and interference of the $n$-th DU, $\mathbf{y}=\left\{y_{1}, \ldots, y_{N}\right\}$, and $y_{n} \geq 0$ is the interference price for the $n$-th DU, $\mu_{n}$ represents the tradeoff between the data rate and the energy cost. In (3), the first, the second and the third terms refer to the data rate, weighted power consumption cost and the interference cost caused to the CU, respectively.

Then, let $G=\left[\mathcal{N},\left\{P_{n}\right\},\left\{\Psi_{n}(\cdot)\right\}\right]$ denotes the noncooperative power allocation game, where $\mathcal{N}=\{1,2, \ldots, N\}, P_{n}=\left[0, p_{\text {max }}\right]$ is the strategy set of each DU, $\Psi_{n}(\cdot)$ is the net utility of the $n$-th DU.

\section{2) The BS (Seller) Side}

The BS charges for the interference caused by the DUs and tries the best to benefit from the interference profit as much as possible. The revenue for the BS and the constraint of the received interference can be expressed as below:

$$
\begin{gathered}
\Gamma_{m}(\mathbf{y})==_{\mathbf{y} \succeq 0} \sum_{n=1}^{N} f_{n m} p_{n} y_{n}, \\
\text { s.t. } \sum_{n=1}^{N} f_{n m} p_{n} \leq \Upsilon .
\end{gathered}
$$

For the seller, in order to protect $\mathrm{CU}$, the optimization problem of BS is to maximize $\Gamma_{m}(\mathbf{y})$ and obtains the optimal $\mathbf{y}$.

The solution of the Stackelberg game can be explained as follows.

\subsection{Analysis of the Stackelberg Equilibrium}

In this subsection, we propose two pricing strategies to maximize the net utility of the DUs and BS. The two pricing methods are uniform pricing strategy and uneven pricing strategy, respectively. It means that the interference prices of the DUs would be equal and unequal. According to different pricing strategies, we analyze the Stackelberg game.

1) The D2D (Buyer) Side

As the buyer, when the interference prices are provided, each DU wants to maximize the net utility by adjusting the transmission power.

When $y_{n}$ is fixed, we can differentiate $\Psi_{n}$ with respect to $p_{n}$. Then, we can get 


$$
\frac{\partial \Psi_{n}}{\partial p_{n}}=\frac{W g_{n}}{\left(I_{n}+p_{n} g_{n}\right) \ln 2}-\mu_{n} X-\varepsilon_{n} f_{n m} y_{n},
$$

where $I_{n}=\sigma_{n}^{2}+p_{B} g_{n m}+\sum_{l \neq n}^{N} p_{l} h_{l n}$.

Based on (7), we can also get

$$
\frac{\partial^{2} \Psi_{n}}{\partial^{2} p_{n}}=-\frac{W g_{n}^{2}}{\left(I_{n}+p_{n} g_{n}\right)^{2} \ln 2}<0 .
$$

According to (7) and (8), we can observe that the net utility function is concave. Therefore, by setting ( 7 ) to be zero, we can get:

$$
p_{n}=\frac{W}{\left(\mu_{n} x+\varepsilon_{n} f_{n m} y_{n}\right) \ln 2}-\frac{I_{n}}{g_{n}} .
$$

Proposition 1. The best response function of the n-th DU is written by

$$
p_{n}=\mathcal{B}\left(\overline{\mathbf{p}_{n}}\right) \text {, }
$$

where

$$
\mathcal{B}\left(\overline{\mathbf{p}_{n}}\right)=\left[\frac{W}{\left(\mu_{n} x+\varepsilon_{n} f_{n m} y_{n}\right) \ln 2}-\frac{I_{n}}{g_{n}}\right]_{0}^{p_{\max }},
$$

where $[\cdot]_{a}^{b}=\max (\min (\cdot, b), a)$ and $\overline{\mathbf{p}_{n}}$ represents the power vector $\left\{p_{1}^{*}, \ldots, p_{n-1}^{*}, p_{n+1}^{*}, \ldots p_{N}^{*}\right\}$ for all the DTs except the $n$-th DT.

According to the (10) and (11), we can observe that the optimal transmission power of one DU will affect the transmission power of other DUs. We can know that for $1 \leq n \leq N, p_{n} \geq 0$. If the transmission power of all the DUs equals zero, then, the price $y_{n}^{h}$ for the $n$-th DU can be expressed by

\section{2) The BS (Seller) Side}

$$
y_{n}^{h}=\frac{W g_{n}}{\sigma_{n}^{2} \varepsilon_{n} f_{n m} \ln 2}-\frac{\mu_{n} x}{\varepsilon_{n} f_{n m}} .
$$

In the following content, we propose two strategies to design the interference price vector.

1. Uniform Pricing Strategy: In this case, the elements of the interference price vector are equal, i.e., $y=y_{1}=\ldots y_{N}$. So, (5) and (6) can reduce to a one-dimensional search problem. If $y$ decreases, the optimal transmission power of the DUs would increase. However, the transmission power of the DUs cannot be larger than the maximum power $p_{\max }$. According to (4) and (11), when all the DUs transmit with the maximum power $p_{\max }$, the uniform interference price would be the lower boundary of the prices for the DUs, it can be written as

$$
y^{l}=\min _{n \in\{1, \ldots, N\}}\left(\frac{W g_{n} /\left(\ln 2 \cdot \varepsilon_{n} f_{n m}\right)}{\left(\sigma_{n}^{2}+p_{B} g_{n m}+\sum_{l \neq n}^{N} p_{\max } h_{l n}\right)}-\frac{\mu_{n} x}{\varepsilon_{n} f_{n m}}\right) .
$$

The upper boundary of the prices can be written as

$$
y^{h}=\max _{n \in\{1, \ldots, N\}}\left(y_{n}^{h}\right) \text {. }
$$


Remark 1: we can know that the optimal interference price $y^{*}$ is between $y^{l}$ and $y^{h}$. Based on the analysis above, we can see that if the interference prices are less than $y^{l}$, DUs transmit with the maximum power and the interference profit function increases when $y$ increases. If the interference prices are larger than $y^{h}$, the DUs cannot pay for it, and the interference profit is equal to zero. However, when $y^{l}<y<y^{h}$, with the increase of the $y$, the transmission power of the DUs will decrease.

\section{Algorithm 1 Uniform Pricing Algorithm}

1 , Set $y=y^{h}$ for all the DUs, $t=0, \epsilon=0.001$

2, Repeat iterations

$t=t+1$

Perform the Algorithm 2, get the optimal power of the DUs.

Calculate the power of the received interference at CU.

if $\Upsilon(t)<\Upsilon$

$$
y(t+1)=y(t)-\epsilon
$$

else $y^{*}=y(t)$.

end if

until $t \geq T_{\max }$ or $y(t+1) \leq y^{l}$.

3, End iterations.

4, Find the maximum interference revenue, the corresponding price is the optimal price $y^{*}$

\section{Algorithm 2 Distributed Power Allocation Algorithm}

1 , Given the price vector, set $p_{n}=0, t=0$.

2, Repeat iterations

$t=t+1$

for $n=1: N$

$p_{n}[t]=\mathcal{B}\left(p_{1}[t-1], \ldots, p_{n-1}[t-1], p_{n+1}[t-1], \ldots, p_{N}[t-1]\right)$

end if

until $(1-\varsigma) \cdot p_{n}[t-1] \leq p_{n}[t] \leq(1+\varsigma) \cdot p_{n}[t-1], n \in \mathrm{N}$, where $\varsigma$ equals to 0.01 .

3 , End iterations

Then, we can set $y=y^{h}$ or $y=y^{l}$ at first in Algorithm 1 to find the optimal solution. $T_{\max }$ is the maximum iteration count.

The most popular solution used for the game is Nash equilibrium [15]. The definition is that a power vector $\mathbf{p}=\left(p_{1}, \ldots, p_{N}\right)$ is a Nash equilibrium of $G=\left[\mathcal{N},\left\{P_{n}\right\},\left\{\Psi_{n}(\cdot)\right\}\right]$, if, for every $n \in \mathcal{N}, \Psi_{n}\left(p_{n}\right) \geq \Psi_{n}\left(p_{n}^{\prime}\right)$ for all $p_{n}^{\prime} \in P_{n}$. In Algorithm 2, a distributed power allocation method is used to obtain the Nash equilibrium for the noncooperative power control game.

Based on [16], we can get that a unique equilibrium exists in $G=\left[\mathcal{N},\left\{P_{n}\right\},\left\{\Psi_{n}(\cdot)\right\}\right]$, and the power allocation algorithm converges. Moreover, the convergence of this algorithm is shown in Fig. 2.

2. Uneven Pricing Strategy: In this strategy, in order to simplify the power allocation and reduce the interactions between all the DUs, we can assume that the interference between the 
DUs can be neglected until we find the optimal solution. It is based on the fact that compared with the interference caused by the BS, the interference between the DUs is quite small. Therefore, according to (10) and (11), we can obtain $\mathcal{B}\left(\overline{\mathbf{p}_{n}}\right)=\left[\frac{W}{\left(\mu_{n} x+\varepsilon_{n} f_{n m} y_{n}\right) \ln 2}-\frac{\sigma_{n}^{2}+p_{B} g_{n m}}{g_{n}}\right]_{0}^{p_{\max }}$, and $\mathcal{B}\left(\overline{\mathbf{p}_{n}}\right)$ is affected just by the channel gains $f_{n m}, g_{n}, g_{n m}$ and the weights $\mu_{n}, \varepsilon_{n}$.

If $\frac{W}{\left(\mu_{n} x+\varepsilon_{n} f_{n m} y_{n}\right) \ln 2}-\frac{\sigma_{n}^{2}+p_{B} g_{n m}}{g_{n}}<0$, i.e., $y_{n}>\frac{W g_{n}}{\left(\sigma_{n}^{2}+p_{B} g_{n m}\right) \varepsilon_{n} f_{n m} \ln 2}-\frac{\mu_{n} x}{\varepsilon_{n} f_{n m}}$, it means that the $n$-th DU stops transmitting. We can set $T(n)=\frac{W g_{n}}{\left(\sigma_{n}^{2}+p_{B} g_{n m}\right) \varepsilon_{n} f_{n m} \ln 2}-\frac{\mu_{n} x}{\varepsilon_{n} f_{n m} \ln 2}$. Without loss generality, we assume $T(1)>\ldots>T(N)$. Therefore, (5) should be converted into several concave problems within different intervals. However, as the optimal transmission power of the DU cannot be larger than $p_{\max }$, it means that $\frac{W}{\left(\mu_{n} x+\varepsilon_{n} f_{n m} y_{n}\right) \ln 2}-\frac{\left(\sigma_{n}^{2}+p_{B} g_{n m}\right)}{g_{n}} \leq p_{\max } \quad$, i.e., $y_{n} \geq \frac{W g_{n}}{\left(\sigma_{n}^{2}+p_{B} g_{n m}+p_{\max } g_{n}\right) \varepsilon_{n} f_{n m} \ln 2}-\frac{\mu_{n} x}{\varepsilon_{n} f_{n m}} \quad$. $\quad$ Then, we set $S(n)=\frac{W g_{n}}{\left(\sigma_{n}^{2}+p_{B} g_{n m}+p_{\max } g_{n}\right) \varepsilon_{n} f_{n m} \ln 2}-\frac{\mu_{n} x}{\varepsilon_{n} f_{n m}}$, where $S(1)>\ldots>S(N)$.

Theorem 1. When $\sum_{n=1}^{N}\left(\frac{W}{\left(\mu_{n} x+\varepsilon_{n} f_{n m} T(n)\right) \ln 2}-\frac{\sigma_{n}^{2}+p_{B} g_{n m}}{g_{n}}\right) \leq \Upsilon<\min \left(\sum_{n=1}^{N}\left(\frac{W}{\left(\mu_{n} x+\varepsilon_{n} f_{n m} z_{n}(0) \ln 2\right.}-\frac{\sigma_{n}^{2}+p_{B} g_{n m}}{g_{n}}\right), U(N)\right)$, The optimal price for the $n$-th DU $y_{n}^{*}$ can be written as

$$
y_{n}^{*}=\left(\frac{\sqrt{\frac{W\left(\mu_{n} x+w \varepsilon_{n} f_{n m}\right) f_{n m}}{\left(\frac{\left(\sigma_{n}^{2}+p_{B} g_{n m}\right) f_{n m}}{g_{n}}+\frac{\Upsilon}{N}\right) \ln 2}}-\mu_{n} x}{\varepsilon_{n} f_{n m}}\right)_{S(n)}^{T(N)} .
$$

When

$\left(\frac{W}{\left(\mu_{1} X+\varepsilon_{1} f_{1 m} T(1)\right) \ln 2}-\frac{\sigma_{1}^{2}+p_{B} g_{1 m}}{g_{1}}\right) \leq \Upsilon<\min \left(\frac{W}{\left(\mu_{1} X+\varepsilon_{1} f_{1 m} T(2)\right) \ln 2}-\frac{\sigma_{1}^{2}+p_{B} g_{1 m}}{g_{1}}, U(1)\right)$, only the 1-th DU can transmit successfully, it is because that the interference prices for other DUs are too high. 


$$
y_{1}^{*}=\left(\frac{\sqrt{\frac{W\left(\mu_{1} x+w \varepsilon_{n} f_{1 m}\right) f_{1 m}}{\left(\frac{\left(\sigma_{1}^{2}+p_{B} g_{1 m}\right) f_{1 m}}{g_{1}}+\frac{\Upsilon}{N}\right) \ln 2}}-\mu_{1} x}{\varepsilon_{1} f_{1 m}}\right)_{S(1)}^{T(1)}
$$

When $\Upsilon<\left(\frac{W}{\left(\mu_{1} x+\varepsilon_{n} f_{1 m} T(1)\right) \ln 2}-\frac{\sigma_{1}^{2}+p_{B} g_{1 m}}{g_{1}}\right)$, all the DUs cannot transmit successfully.

The procedure of the uneven pricing strategy and the power allocation can be obtained in Algorithm 3.

Proof. Please see Appendix A.

Remark 2: As we could notice, the solution obtained in our scheme would be useful in practice. In both strategies, when the communication quality of the CUs is good and the need of D2D communication increases, we can increase $\Upsilon$ or decrease interference prices to addmit more DUs. Otherwise, if the cellular network traffic is large or the QoS of the CUs is low, we can protect the cellular networks by increasing the interference price or decreasing $\Upsilon$.

Remark 3: Based on the analysis above, our proposed strategies all rely on the local CSI, and the additional feedback is that BS should send the interference price to DUs. Compared to the comparison schemes in the simulations, the proposed schemes achieve higher energy efficiency with only a moderate increase of feedback overhead.

Algorithm 3 Uneven Pricing Algorithm

1, Initialization: BS collects the CSI in the networks, then we can initialize the power vector.

2, BS calculates the intervals $[T(1), \ldots, T(N)]$ and $[S(1), \ldots, S(N)]$, computes the limit range based on the given $\Upsilon$.

3, After the DUs get the interference prices set by BS according to Theorem 1, the optimal power of the DUs can be got by performing Algorithm 2.

4, BS calculates the interference profit.

\section{Numerical Results}

In this section, we present various simulations in MATLAB to further verify the derived analytical results. As the spectrums used by CUs are orthogonal, we just assume that there is one BS, one CU and many DUs, where CU and DUs are randomly distributed with a coverage radius $500 \mathrm{~m}$. Each CU shares the same resource with 40 DUs. It is assumed that all the users equipped with single antenna. The model for path-loss is designed as $H=G_{D} \cdot(d)^{-\alpha} h$, where $H$ is an element of the channel vector, $h$ is the Gaussian channel coefficient with the distribution $\mathcal{N}(0,1) . \alpha$ is a path loss exponent, $d$ is the distance between the two nodes, $G_{D}$ is the channel gain at a distance of $1 \mathrm{~m}$. Without loss of generality, we let the weights $\varepsilon_{n}=2$, $x=1$. We assume the maximum transmission power for all DUs is equal. Unless otherwise stated, these parameters in Table 1 stay the same in the following simulations. 
Table 1. SIMULATION PARAMETERS

\begin{tabular}{|c|c|}
\hline Parameters & Value \\
\hline \hline Radius of the cell & $500 \mathrm{~m}$ \\
\hline The distance between each DT and DR in a pair & $10 \mathrm{~m}$ \\
\hline Path loss exponent for D2D links & 2 \\
\hline Path loss exponent for cellular links & 3 \\
\hline Downlink Bandwith $W$ & $3 \mathrm{MHZ}$ \\
\hline Numbers of DUs $N$ & 20 \\
\hline Maximum transmission power of each DU $p_{\max }$ & $20 \mathrm{dBm}$ \\
\hline The transmission power of the BS $p_{B}$ & $46 \mathrm{dBm}$ \\
\hline The interference power margin of CU $\Upsilon$ & $-10 \mathrm{~dB}$ \\
\hline AWGN noise power & $-174 \mathrm{dBm} / \mathrm{HZ}$ \\
\hline For $n \in\{1, \ldots, N\}, \varepsilon_{n}$ & 2 \\
\hline$X$ & 1 \\
\hline
\end{tabular}

\section{A. The convergence for Algorithm 2}

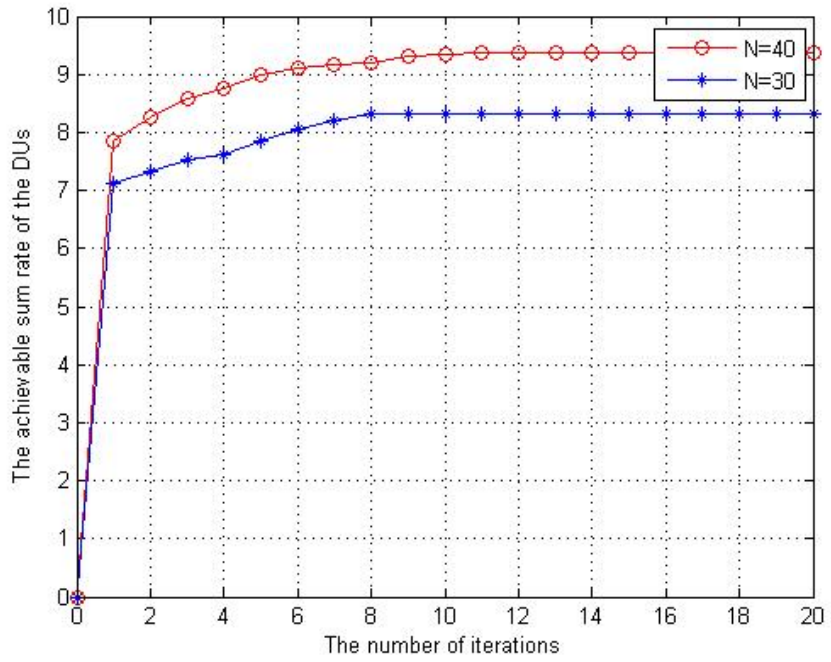

Fig. 2. The convergence behavior for data rates of D2D transmission in Algorithm 2.

Fig. 2 shows the convergence behavior of the D2D networks during the non-cooperative competition. We can see that when the number of the DUs is different, the sum rate of the D2D networks still converges after several iterations. 


\section{B. The revenue of the $\mathrm{BS}$ in Stackelberg game}

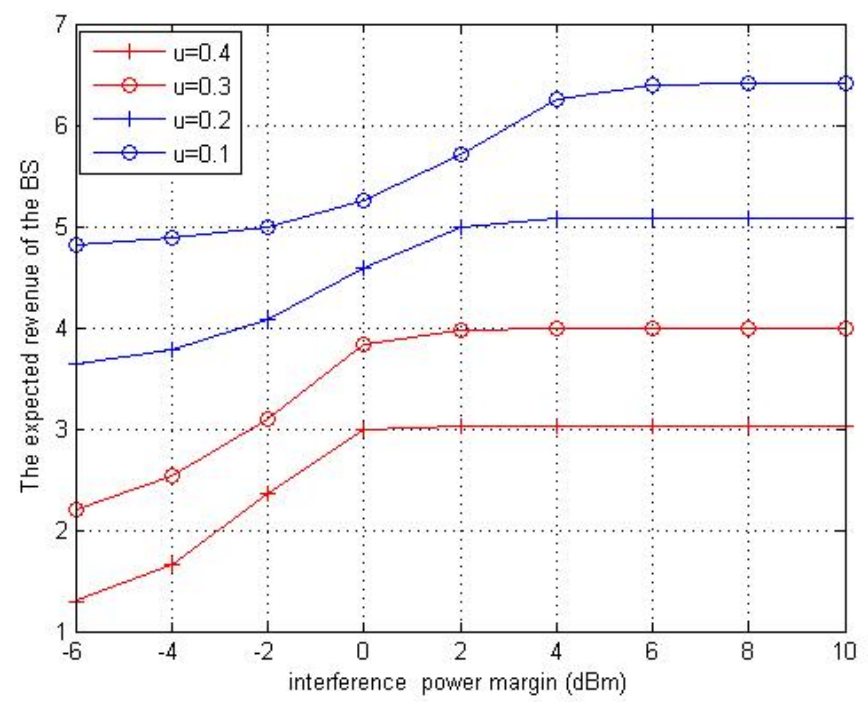

Fig. 3. The revenue of the cellular networks with the various interference power constraint.

In Fig. 3, we set the weight $\mu=\mu_{1}=\ldots=\mu_{N}$. Take the uneven pricing strategy as an example. And we can observe that the interference profit of the cellular networks decreases linearly with the weight $\mu$. The reason is that due to the power used to allocate resource, there is a tradeoff between the transmission power and the power consumption in the net utility function of the D2D networks. Then, with the increase of $\mu$, the interference price of the BS would decrease. As the interference margin reaches a value, the optimal price and optimal power become constant. As the interference control works, the expected revenue also becomes constant.

\section{The energy efficiency of the D2D networks}

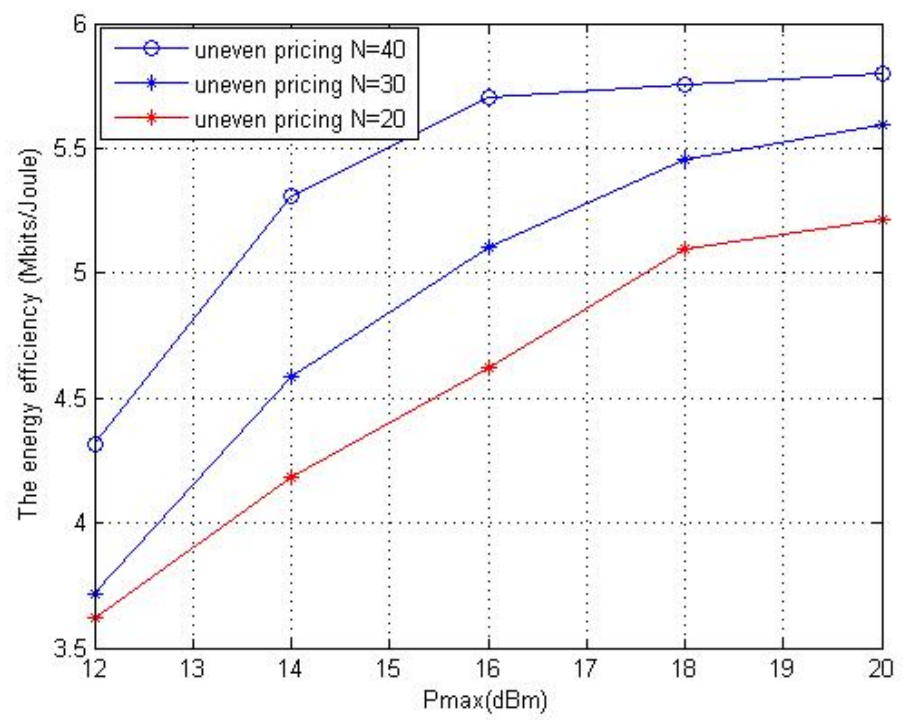

Fig. 4. The energy efficiency of the D2D networks with the uneven pricing strategy. 
In Fig. 4, we show the EE comparison among the different number of the D2D pairs. Take the uneven pricing strategy as an example. The energy efficiency is drawn in terms of the maximum transmission power of the DUs. We set the number of the DUs which share the same resource with one CU is $N=20, N=30$ and $N=40$. With our uneven pricing scheme, increasing the number of the D2D pairs can improve the sum rate of the system faster than the power consumption, which eventually increases the energy efficiency. On the one hand, as the number of the D2D pairs increases, the sum rate of the DUs can be improved. On the other hand, by using our proposed scheme, the sum rate of the D2D networks can be further improved with the power allocation.

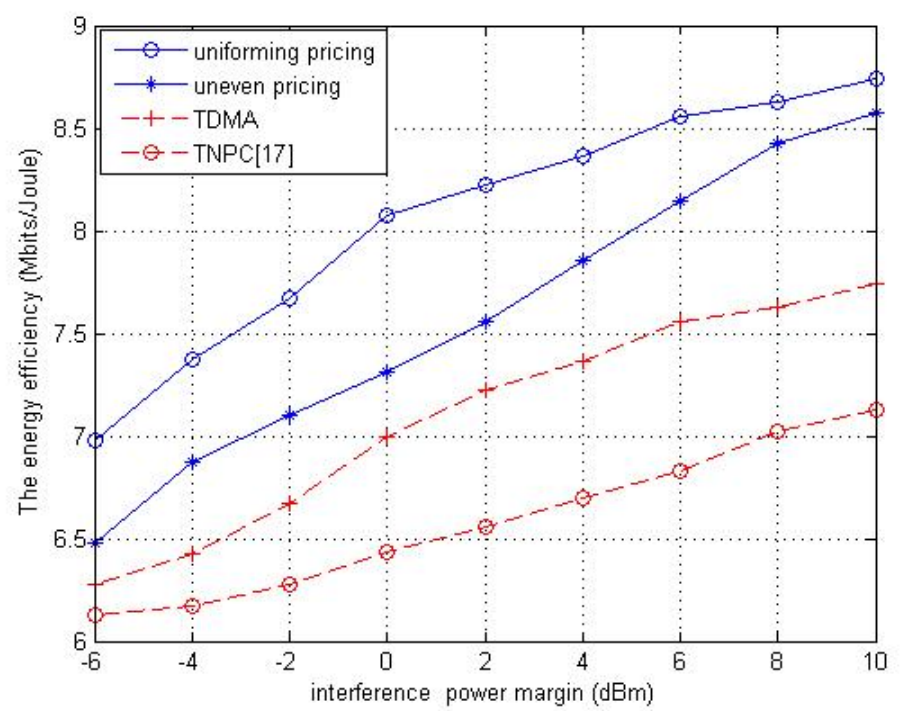

Fig. 5. The energy efficiency of the D2D networks.

In Fig. 5, we compare the EE of our proposed two strategies and the traditional schemes without pricing for interference control. The one is the scheme with TDMA, the other scheme is with the traditional power control scheme (TPC) [17]. In the TDMA scheme, based on the principle of fairness, the DUs can transmit in a round-robin fashion at different time instances, and the transmission power is $\min \left(p_{\max }, \Upsilon / f_{n m}\right)$. In the TNPC scheme, the DUs transmit with the maximum power. Only when the power of the interference received by corresponding CU is not larger than the interference margin, the DUs are allowed to transmit signals. We can see that the EE of our proposed scheme can be superior to the traditional schemes, and it is because that in consideration of the states of the all users, our schemes can allocate the power resource better. In TNPC scheme, when the interference received is large, DUs would stop transmitting. So, the TNPC scheme has the worst performance. For the uneven pricing strategy, the interference prices for each DU are different, and the best performance can be achieved with this strategy. 


\section{The sum rate of the DUs}

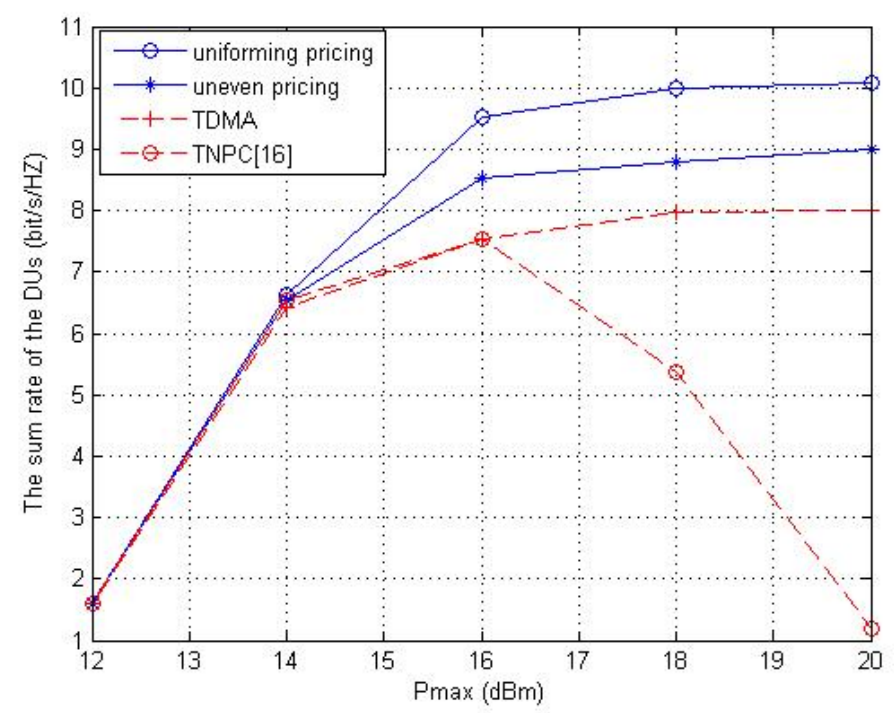

Fig. 6. The sum rate of the D2D networks.

In the Fig. 6, we can observe that when the maximum transmission power of the DUs is small, the sum rate of the TNPC, the TDMA scheme and our scheme are overlapped. It is because that the transmission power of the DUs is equal. With the increase of the maximum transmission power of the DUs, the sum rate of our two strategies and the TDMA scheme increase, then the values of these schemes are bounded. The reason is that when the maximum transmission power is large enough, the interference constraint plays an important role in the game. As for the TNPC scheme, the sum rate decreases when the maximum transmission power of the DUs increases. It is because that when maximum transmission power increases, the interference power received by the CU is larger than the interference margin, and several DUs may stop transmitting.

\section{Conclusion}

In this paper, we have introduced a scheme to maximize the EE of the DUs and the interference profit of BS with the constraint of the QoS for the CU. We use the Stackelberg game based on the pricing mechanism to model the interactions between the BS and the DUs. Also, we have proposed two pricing strategies, i,e., uniform and uneven pricing strategies to maximize the interference profit. By using these strategies, for the uneven pricing algorithm, a backward induction method is used to get the solution in the Stackelberg equilibrium with the interference power constraint. For the uniform pricing algorithm, the boundary of the interference prices can be obtained, we can know the value of the initial price. Adopting our strategies, the EE of the networks can be further improved. Combined with results of the currently, we are still working on rigorous theoretical analysis for the second strategy in consideration of interference between different DUs, and the analysis in the general system model will be shown in our future work. 


\section{Appendix A: Proof of Theorem 1}

Take the situation $\mathbf{y} \leq T(N)$, all the D2D pairs can communicate. The optimal interference prices can be obtained by the Karush-Kuhn-Tucker (KKT) conditions. The Lagrangian associated with the given interference problem can be written as [18].

$$
\begin{gathered}
L(\mathbf{y}, w, \alpha, \beta)=\sum_{n=1}^{N} f_{n m} y_{n} Q_{n}-w\left(\sum_{n=1}^{N} f_{n m} Q_{n}-\Upsilon\right)-\alpha\left(y_{n}-T(N)\right)+\beta\left(y_{n}-S(n)\right), \\
\sum_{n=1}^{N} f_{n m} Q_{n}-\Upsilon \leq 0, \\
S(n)<y_{n}<T(N),
\end{gathered}
$$

where $w, \alpha, \beta$ are the Lagrange multipliers, $Q_{n}=\frac{W}{\left(\mu_{n} x+\varepsilon_{n} f_{n m} y_{n}\right) \ln 2}-\frac{\sigma_{n}^{2}+p_{B} g_{n m}}{g_{n}}$, $S(n)=\frac{W g_{n}}{\left(\sigma_{n}^{2}+p_{B} g_{n m}+p_{\max } g_{n}\right) \varepsilon_{n} f_{n m} \ln 2}-\frac{\mu_{n} x}{\varepsilon_{n} f_{n m}}$. According to (18), when $\Upsilon<\sum_{n=1}^{N} f_{n m} Q_{n}$, the QoS of CU can not be satisfied.

Then, we can differentiate $L(\mathbf{y}, w, \alpha, \beta)$ with respect to $y_{n}$,

$$
\frac{\partial L(\mathbf{y}, w, \alpha, \beta)}{y_{n}}=\frac{f_{n m} W\left(\mu_{n} x+w \varepsilon_{n} f_{n m}\right)}{\left(\mu_{n} x+\varepsilon_{n} f_{n m} y_{n}\right)^{2} \ln 2}-\frac{f_{n m}\left(\sigma_{n}^{2}+p_{B} g_{n m}\right)}{g_{n}}-\alpha+\beta=0 .
$$

If for $n \in\{1, \ldots, N\}, \alpha\left(y_{n}-T(N)\right)=0$ and $\alpha \neq 0$, then, $\beta=0$. According to the (20), we can get

$$
\frac{f_{n m} W\left(\mu_{n} x+w \varepsilon_{n} f_{n m}\right)}{\left(\mu_{n} x+\varepsilon_{n} f_{n m} y_{n}\right)^{2} \ln 2}-\frac{f_{n m}\left(\sigma_{n}^{2}+p_{B} g_{n m}\right)}{g_{n}}-\alpha=0 .
$$

Substituting (21) into (18), we can get

$$
\sum_{n=1}^{N} \alpha-\Upsilon=0
$$

Then, $\alpha=\frac{\Upsilon}{N}$.

If for $n \in\{1, \ldots, N\}, \beta\left(y_{n}-S(n)\right)=0$ and $\beta \neq 0$, then, $\alpha=0$. Therefore, $\beta=-\frac{\Upsilon}{N}$ which contradicts $\beta>0$. Based on the analysis above, we can get $\alpha=\frac{\Upsilon}{N}$ and $\beta=0$. For $w$, we can calculate this multiplier by the binary search algorithm.

According to (17), by setting $\frac{\partial L(\mathbf{y}, w, \alpha, \beta)}{\partial y_{n}}$ to zero, we can get 


$$
y_{n}=z_{n}(w)=\left(\frac{\sqrt{\left(\frac{W\left(\mu_{n} x+w \varepsilon_{n} f_{n m}\right) f_{n m}}{\left(\sigma_{n}^{2}+p_{B} g_{n m}\right) f_{n m}}+\frac{\Upsilon}{N}\right) \ln 2}-\mu_{n} x}{\varepsilon_{n} f_{n m}}\right)^{T(N)},
$$

where $(\cdot)_{a}^{b}=\min (\max (\cdot, a), b)$.

$$
\text { If } \sum_{n=1}^{N}\left(\frac{W}{\left(\mu_{n} x+\varepsilon_{n} f_{n m} z_{n}(0) \ln 2\right.}-\frac{\left(\sigma_{n}^{2}+p_{B} g_{n m}\right)}{g_{n}}\right) \leq \sum_{n=1}^{N}\left(\frac{W}{\left(\mu_{n} x+\varepsilon_{n} f_{n m} S(N) \ln 2\right.}-\frac{\left(\sigma_{n}^{2}+p_{B} g_{n m}\right)}{g_{n}}\right)
$$

and $\Upsilon \geq \sum_{n=1}^{N}\left(\frac{W}{\left(\mu_{n} x+\varepsilon_{n} f_{n m} z_{n}(0) \ln 2\right.}-\frac{\left(\sigma_{n}^{2}+p_{B} g_{n m}\right)}{g_{n}}\right), w=0, y_{n}=z_{n}(0)$;

$$
\text { If } \sum_{n=1}^{N}\left(\frac{W}{\left(\mu_{n} x+\varepsilon_{n} f_{n m} z_{n}(0) \ln 2\right.}-\frac{\left(\sigma_{n}^{2}+p_{B} g_{n m}\right)}{g_{n}}\right)>\sum_{n=1}^{N}\left(\frac{W}{\left(\mu_{n} x+\varepsilon_{n} f_{n m} S(1) \ln 2\right.}-\frac{\left(\sigma_{n}^{2}+p_{B} g_{n m}\right)}{g_{n}}\right)
$$

and $\Upsilon \geq \sum_{n=1}^{N}\left(\frac{W}{\left(\mu_{n} x+\varepsilon_{n} f_{n m} S(N) \ln 2\right.}-\frac{\left(\sigma_{n}^{2}+p_{B} g_{n m}\right)}{g_{n}}\right), y_{n}=S(n)$;

$\sum_{n=1}^{N}\left(\frac{W}{\left(\mu_{n} X+\varepsilon_{n} f_{n m} T(N)\right) \ln 2}-\frac{\left(\sigma_{n}^{2}+p_{B} g_{n m}\right)}{g_{n}}\right) \leq \Upsilon<\min \left(\sum_{n=1}^{N}\left(\frac{W}{\left(\mu_{n} X+\varepsilon_{n} f_{n m} z_{n}(0) \ln 2\right.}-\frac{\sigma_{n}^{2}+p_{B} g_{n m}}{g_{n}}\right), U(N)\right)$, where $w \neq 0, U(k)=\sum_{n=1}^{N}\left(\frac{W}{\left(\mu_{n} x+\varepsilon_{n} f_{n m} S(k) \ln 2\right.}-\frac{\sigma_{n}^{2}+p_{B} g_{n m}}{g_{n}}\right)$, we can find the $y_{n}$ meets the KKT conditions. Then, we can use the binary search algorithm to find the Lagrange multiplier $w[19]$;

If $\Upsilon<\sum_{n=1}^{N}\left(\frac{W}{\left(\mu_{n} x+\varepsilon_{n} f_{n m} T(N)\right) \ln 2}-\frac{\sigma_{n}^{2}+p_{B} g_{n m}}{g_{n}}\right)$, we cannot get the optimal transmission power.

So, we can use the same method to find different optimal power in different intervals. Then, according to the analysis and the binary search algorithm above, the optimal prices and the Lagrange multiplier $w$ can be obtained. 


\section{References}

[1] K. Doppler, M. Rinne, C. Wijting, C. Ribeiro, and K. Hugl, "Device-to-Device communication as an Underlay to LTE-advanced networks,” Commun. Mag., vol. 7, no. 12, pp. 42-49, Dec. 2009. Article (CrossRefLink)

[2] G. Fodor, E. Dahlman, G. Mildh, S. Parkvall, N. Reider, G. Miklòs, and Z. Turányi, "Design aspects of network assisted device-to-device communications,” IEEE Commun. Mag., vol. 50, no. 3, pp. 170-177, Mar. 2012. Article (CrossRef Link)

[3] H. Gao, T. Lv, D. Fang, S. Yang, and C. Yuen, "Limited Feedback-Based Interference Alignment for Interfering Multi-Access Channels,” IEEE Commun. lett., vol. 18, no.4, pp. 540-543, Apr. 2014. Article (CrossRef Link)

[4] X. Chen, L. Chen, M. Zeng, X. Zhang, and D. Yang, "Downlink resource allocation for Device-to-Device communication underlaying cellular networks,” in Proc. IEEE PIMRC, 2012, pp.232-237. Article (CrossRef Link)

[5] E. Yaacoub, H. Ghazzai, M. S. Alouini, and A. Abu-Dayya, "Achieving energy efficiency in LTE with joint D2D communications and green networking techniques,” in IWCM, July. 2013. Article (CrossRef Link)

[6] C. H. Yu, K. Doppler, C. B. Ribeiro, and O. Tirkkonen, "Resource Sharing Optimization for Device-to-Device Communication Underlaying Cellular Networks,” IEEE TWC, vol. 10, no. 8, pp. 2752-2763, August. 2011. Article (CrossRef Link)

[7] Wikipedia. Article (CrossRef Link)

[8] J. Huang, R. A. Berry, and M. L. Honig, "Distributed interference compensation for wireless networks,” IEEE JASC, vol. 24, no. 5, pp. 1074-1084, May. 2006. Article (CrossRef Link)

[9] Y. He, X. Luan, J. Wang, M. Feng, and J. Wu, "Power allocation for D2D communications in heterogeneous networks," in Proc. of ICACT, pp. 1041- 1044, 2014. Article (CrossRef Link)

[10] Y. Zhang, Y. Xu, M. Gao, Q. Zhang, H. Li, I. Ahmad, and Z. Feng, "Resource management in device-to-device underlaying cellular network,” in Proc. of WCNC, pp. 1631-1636, 2015. Article (CrossRef Link)

[11] Z. Hasan, G. Bansal, E. Hossain, and V. K. Bhargava, "Energy-efficient power allocation in OFDM-based cognitive radio systems: A risk-return model,” IEEE TWC, December. 2009. Article (CrossRef Link)

[12] C. Xiong, G. Y. Li, S. Zhang, Y. Chen, and S. Xu, "Energy-Efficient Resource Allocation in OFDMA Networks,” IEEE Trans. on Communications, vol. 60, no. 12 pp. 3767-3778, Dec. 2012. Article (CrossRef Link)

[13] S. Bu, F. R. Yu, and H. Yanikomeroglu, "Interference-Aware Energy-Efficient Resource Allocation for OFDMA-Based Heterogeneous Networks With Incomplete Channel State Information,” IEEE TVT. vol. 64, no. 3, pp. 1036-1050, March. 2015. Article (CrossRef Link)

[14] J. Zhang and Q. Zhang, "Stackelberg game for utility-based cooperative cognitive radio networks," in Proc. of MobiHoc'09, pp. 23-32, 2009. Article (CrossRef Link)

[15] J. F. Nash, “Non-cooperative games,” Ann. Math., vol. 54, pp. 289-295, 1951. Article (CrossRef Link)

[16] C. U. Saraydar, N. B. Mandayam, and D. J. Goodman, "Efficient power control via pricing in wireless data networks,” IEEE Trans. on Comm., vol. 50, no. 2, Feb. 2002. Article (CrossRef Link)

[17] H. ElSawy and E. Hossain, "Analytical modeling of mode selection and power control for underlay D2D communication in cellular networks,” IEEE Trans. Commun., vol. 62, no. 11, pp. 4147-4161, Nov. 2014. Article (CrossRef Link)

[18] S. Boyd and L. Vandenberghe, "Convex Optimization. Cambridge,” U.K. Cambridge Uni. Press, 2004. Article (CrossRef Link)

[19] W. Yu and J. M. Cioffi, "On constant power water-filling,” in Proc of ICC, vol. 6, pp. 1655-1669, 2001. Article (CrossRef Link) 


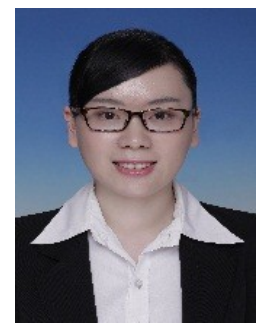

Li Xiaomeng received the B.E degree in Telecommunications engineering from Beijing University of Technology in 2014. She is currently pursuing the M.S. degree in Beijing University of Post and Telecommunications, Beijing, China. Her research interests include interference management in wireless communications.

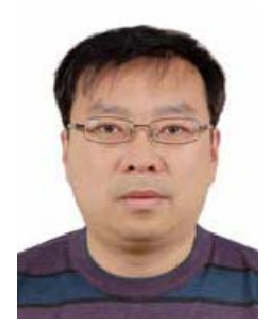

Tiejun Lv received the M.S. and Ph.D. degrees in electronic engineering from the University of Electronic Science and Technology of China (UESTC), Chengdu, China, in 1997 and 2000, respectively. From January 2001 to January 2003, he was a Postdoctoral Fellow with Tsinghua University, Beijing, China. In 2005, he became a Full Professor with the School of Information and Communication Engineering, Beijing University of Posts and Telecommunications (BUPT). From September 2008 to March 2009, he was a Visiting Professor with the Department of Electrical Engineering, Stanford University, Stanford, CA, USA. He is the author of more than 80 published journal papers and 170 conference papers on the physical layer of wireless mobile communications. His current research interests include signal processing, communications theory and networking. He was the recipient of the Program for New Century Excellent Talents in University Award from the Ministry of Education, China, in 2006. He received the Nature Science Award in the Ministry of Education of China for the hierarchical cooperative communication theory and technologies in 2015. 